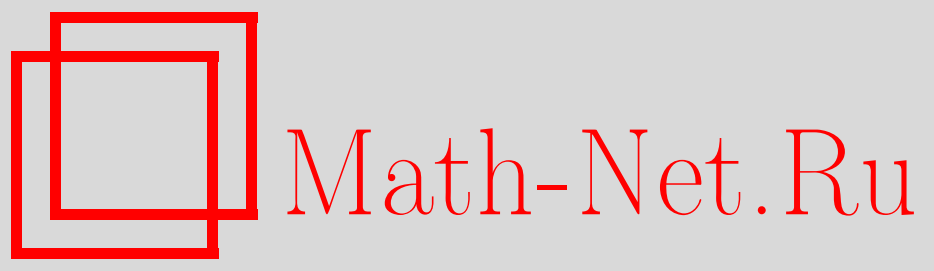

Д. Н. Макаров, В. И. Матвеев, Ионизация в двухмодовом квантованном электромагнитном поле, ТМ $\Phi, 2017$, том 191, номер 1, 25-33

DOI: https://doi.org/10.4213/tmf9157

Использование Общероссийского математического портала Math-Net.Ru подразумевает, что вы прочитали и согласны с пользовательским соглашением http://www . mathnet.ru/rus/agreement

Параметры загрузки:

IP : 35.174 .16 .151

26 апреля 2023 г., 15:46:47

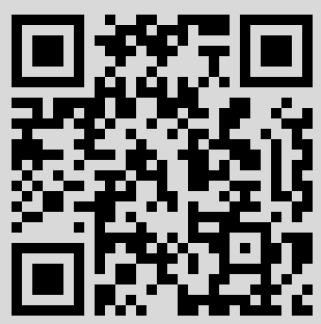




\title{
ИОНИЗАЦИЯ В ДВУХМОДОВОМ КВАНТОВАННОМ ЭЛЕКТРОМАГНИТНОМ ПОЛЕ
}

\begin{abstract}
Найдено аналитическое решение уравнения Шредингера для электрона в двухмодовом квантованном электромагнитном поле. Полученное решение позволяет рассчитывать спектры фотоэлектронов и скорости ионизации атомов в сильных электромагнитных полях.
\end{abstract}

Ключевые слова: квантованное электромагнитное поле, двухмодовое электромагнитное поле, ионизация, уравнение Шредингера, аналитическое решение, фотон, атом.

DOI: https://doi.org/10.4213/tmf9157

\section{1. ВВЕДЕНИЕ}

Исследование ионизации атома электромагнитным полем является важной частью современной физики. Несмотря на достаточно длительный период изучения этого процесса [1]--[3], теория ионизации атома активно развивается в настоящее время [4]. Такое развитие, в первую очередь, связано с созданием мощных лазеров, напряженность поля которых может быть много больше значений напряженности атомных полей (см. [5], где $\epsilon \sim 10^{12} \mathrm{~B} \mathrm{~cm}^{-1}$ ), и проведением численных расчетов. Кроме того, в настоящее время активно развиваются методы генерации ультракоротких импульсов, длительность которых уже приблизилась к аттосекундным масштабам (длительность импульса $\sim 10^{-18} \mathrm{c}$ ), а также исследуется их взаимодействие с веществом [6]. В теоретическом описании взаимодействия электромагнитного поля с атомами вещества в основном используется классическое электромагнитное поле [1], [4], при этом не учитываются квантовые свойства электромагнитного поля. Развитие квантовой теории ионизации (атом и электромагнитное поле неклассические) является фундаментальной задачей современной физики, поскольку может привести к появлению совершенно новых, неизученных явлений. Такое развитие стимулировалось созданием теории неклассических состояний электромагнитного поля и их экспериментальным обнаружением [7], [8].

Работа выполнена в рамках КГЗ Министерства образования и науки РФ (N 3.1726.2014/K), а также при частичной поддержке РФФИ (грант № 15-02-01894) и стипендии Президента РФ (СП-1800.2015.1).

* Северный (Арктический) федеральный университет им. М. В. Ломоносова, Архангельск, Россия. E-mail: makarovd0608@yandex.ru, mezon98@mail.ru 
В настоящей работе рассмотрено взаимодействие сильного двухмодового квантованного электромагнитного поля с атомом. В реальности взаимодействие атома с двухмодовым элекромагнитным полем можно представить как взаимодействие атома в поле двух скрещенных лазерных полей. Если напряженность двухмодового электромагнитного поля много больше атомного, то электрон в атоме можно считать свободным, что позволяет получить волновую функцию такой системы в аналитическом виде. Исследование уравнения Шредингера для атома в квантованном двухмодовом электромагнитном поле является новым и достаточно интересным не только с прикладной, но и с фундаментальной точки зрения, поскольку влияние двухмодового поля на электрон невозможно описать в рамках одномодового поля в случае сильных электромагнитных полей. Ионизация, возникающая при взаимодействии одномодового сильного электромагнитного поля с атомом, была рассмотрена в работе [9], где показано, что полное, квантово-механическое рассмотрение приводит к новым эффектам в фотоионизации. В работе [10] исследована динамика модельной атомной системы в одномодовом квантованном поле. Отметим, что впервые похожая задача в случае одномодового электромагнитного поля для уравнения Дирака была решена в [11], а позднее в работе [12]. Нерелятивистское рассмотрение для одномодового электромагнитного поля впервые было выполнено в работе [13]. Следует отметить, что работа [9] близка к нашей, отличие только в том, что у нас электромагнитное поле двухмодовое. На первый взгляд может показаться, что введение дополнительной моды не сильно отразится на поведении атомной системы в сильном электромагнитном поле. Нами показано, что наличие второй моды приводит к существенному изменению волновой функции системы “электрон в атоме + электромагнитное поле", что делает невозможным описание поведения такой системы в терминах волновой функции одномодового поля. Поиск волновых функций для таких систем - достаточно сложная задача, основной метод решения ее состоит в диагонализации гамильтониана, что и было сделано нами в рассматриваемом случае. Некоторые общие вопросы, связанные с теорией поведения таких квантовых систем, можно найти в [7]--[11].

Изучение процессов фотоионизации в квантованном электромагнитном поле затруднено по сравнению с классическим электромагнитным полем, поскольку уравнения Шредингера в первом случае значительно сложнее, чем во втором. Известно, что даже в дипольном случае не существует аналитической волновой функции электрона в атоме при взаимодействии с классическим электромагнитным полем. Численное решение уравнения Шредингера в случае классического электромагнитного поля достаточно сложное и в сильных электромагнитных полях не приводит к хорошим численным результатам [4]. Поэтому поиск аналитических решений для квантованного электромагнитного поля является актуальным.

\section{2. ПОСТАНОВКА ЗАДАЧИ}

Рассмотрим взаимодействие двухмодового электромагнитного поля с электроном в атоме. Уравнение Шредингера в этом случае имеет вид

$$
i \hbar \frac{\partial \Psi}{\partial t}=\left\{\frac{1}{2 m_{e}}\left(-i \hbar \frac{\partial}{\partial \mathbf{r}}+\frac{e}{c} \widehat{\mathbf{A}}\right)^{2}+\widehat{H}_{f}+U(\mathbf{r})\right\} \Psi
$$


где $m_{e},-e$ - масса и заряд электрона соответственно,

$$
\widehat{H}_{f}=\sum_{\mathbf{k}, \mathbf{u}} \hbar \omega\left(\hat{a}_{\mathbf{k}, \mathbf{u}}^{+} \hat{a}_{\mathbf{k}, \mathbf{u}}+\frac{1}{2}\right)
$$

- гамильтониан электромагнитного поля, $U(\mathbf{r})$ - атомный потенциал, $\hat{a}_{\mathbf{k}, \mathbf{u}}^{+}$и $\hat{a}_{\mathbf{k}, \mathbf{u}}-$ операторы рождения и уничтожения фотонов с волновым вектором $\mathbf{k}$ и поляризацией $\mathbf{u}$, а векторный потенциал

$$
\widehat{\mathbf{A}}=\sum_{\mathbf{k}, \mathbf{u}} \sqrt{\frac{2 \pi c^{2} \hbar}{\omega V_{f}}}\left(\mathbf{u} e^{i(\omega t-\mathbf{k r})} \hat{a}_{\mathbf{k}, \mathbf{u}}+\mathbf{u}^{*} e^{-i(\omega t-\mathbf{k r})} \hat{a}_{\mathbf{k}, \mathbf{u}}^{+}\right) .
$$

Суммирование здесь и выше ведется по всем возможным значениям волнового вектора $\mathbf{k}$ и поляризации $\mathbf{u} . \mathrm{B}$ случае двухмодового электромагнитного поля $\sum_{\mathbf{k}, \mathbf{u}}$ заменится на сумму двух слагаемых, где у первой моды поля волновой вектор $\mathbf{k}_{1}$ с поляризацией $\mathbf{u}_{1}$, а у второй моды волновой вектор $\mathbf{k}_{2}$ с поляризацией $\mathbf{u}_{2}$. Далее, для удобства, перейдем в атомную систему единиц $\hbar=1, m_{e}=1, e=1$. Теперь рассмотрим выражение (1) в дипольном приближении. В этом приближении для двухмодового электромагнитного поля векторный потенциал, выраженный через полевые переменные, имеет вид

$$
\widehat{\mathbf{A}}=a_{1} \mathbf{u}_{1} q_{1}+a_{2} \mathbf{u}_{2} q_{2},
$$

где $a_{1}=\sqrt{4 \pi c^{2} / \omega_{1} V_{f}}, a_{2}=\sqrt{4 \pi c^{2} / \omega_{2} V_{f}}$, а $\omega_{1}, \omega_{2}$ - частоты первой и второй мод соответственно (будем условно считать $\omega_{2}>\omega_{1}$, если частоты полей разные), $V_{f}-$ объем квантования, а $q_{1}, q_{2}$ - полевые переменные первой и второй мод соответственно. В итоге выражение (1) примет вид

$$
\begin{aligned}
i \frac{\partial \Psi}{\partial t}= & \left\{\frac{1}{2}\left(-i \frac{\partial}{\partial \mathbf{r}}+\beta_{1} \mathbf{u}_{1} q_{1}+\beta_{2} \mathbf{u}_{2} q_{2}\right)^{2}+\right. \\
& \left.+\frac{\omega_{1}}{2}\left(q_{1}^{2}-\frac{\partial^{2}}{\partial q_{1}^{2}}\right)+\frac{\omega_{2}}{2}\left(q_{2}^{2}-\frac{\partial^{2}}{\partial q_{2}^{2}}\right)+U(\mathbf{r})\right\} \Psi,
\end{aligned}
$$

где $\beta_{1}=\sqrt{4 \pi / \omega_{1} V_{f}}, \beta_{2}=\sqrt{4 \pi / \omega_{2} V_{f}}$. Далее будем считать, что электромагнитное поле настолько сильное, что потенциалом $U(\mathbf{r})$ можно пренебречь. В итоге нам надо решить стационарное уравнение Шредингера $\widehat{H} \Psi=E \Psi$, где

$$
\widehat{H}=\frac{1}{2}\left(-i \frac{\partial}{\partial \mathbf{r}}+\beta_{1} \mathbf{u}_{1} q_{1}+\beta_{2} \mathbf{u}_{2} q_{2}\right)^{2}+\frac{\omega_{1}}{2}\left(q_{1}^{2}-\frac{\partial^{2}}{\partial q_{1}^{2}}\right)+\frac{\omega_{2}}{2}\left(q_{2}^{2}-\frac{\partial^{2}}{\partial q_{2}^{2}}\right) .
$$

Например, если считать, что второго поля нет $\left(\beta_{2}=0\right.$ и $\left.\omega_{2}=0\right)$, то получим выражение, рассмотренное в работе [9] для одномодового поля.

Скорость ионизации $w$ можно рассчитать по формуле [1]

$$
w=2 \pi \int\left|T_{\mathrm{f}, \mathrm{i}}\right|^{2} \delta\left(E_{\mathrm{f}}-E_{\mathrm{i}}\right) d k,
$$

где $T_{\mathrm{f}, \mathrm{i}}$ - матричный элемент перехода системы из начального состояния с энергией $E_{\mathrm{i}}$ в конечное состояние с энергией $E_{\mathrm{f}}$. В начальном состоянии мы будем 
пренебрегать влиянием электромагнитного поля на волновую функцию электрона в атоме, а в конечном состоянии будем точно учитывать взаимодействие электрона с электромагнитным полем в континууме, но при этом пренебрегать взаимодействием с атомным потенциалом.

Таким образом, нужно решить уравнение Шредингера с гамильтонианом (5), после этого по формуле (6) можно рассчитать скорость ионизации.

\section{3. РЕШЕНИЕ УРАВНЕНИЯ ШРЕДИНГЕРА}

Рассмотрим стационарное уравнение Шредингера с гамильтонианом (5). Представим рассматриваемое дифференциальное уравнение в виде

$$
\widehat{H}^{\prime} \Psi^{\prime}=E \Psi^{\prime}
$$

где $\widehat{H}^{\prime}=\widehat{S} \widehat{H} \widehat{S}^{-1}, \Psi^{\prime}=\widehat{S} \Psi$, а $\widehat{S}$ - некоторый унитарный оператор, $\widehat{S}^{-1}$ - оператор, обратный $\widehat{S}\left(\widehat{S}^{-1} \widehat{S}=1\right)$. Найдя решение уравнения Шредингера (7), получим искомую волновую функцию при обратном преобразовании $\Psi=\widehat{S}^{-1} \Psi^{\prime}$. Далее введем такую систему координат, где по оси $z$ направлен некоторый вектор $\mathbf{S}$, а в плоскости $x O z$ лежит вектор $\boldsymbol{\Sigma}$, где векторы $\mathbf{S}$ и $\boldsymbol{\Sigma}$ определены ниже. Решение будем искать в виде $\Psi=\widehat{S}^{-1} \Psi^{\prime}$, где оператор

$$
\begin{aligned}
\widehat{S}= & \exp \left\{i \xi_{2} \frac{\partial}{\partial x} \frac{\partial}{\partial q_{2}}+i \lambda_{1} \frac{\partial}{\partial z} \frac{\partial}{\partial q_{2}}+i \xi_{1} \frac{\partial}{\partial z} \frac{\partial}{\partial q_{1}}\right\} \exp \left\{-i \lambda_{2} x \frac{\partial}{\partial z}\right\} \times \\
& \times \exp \left\{-i \xi q_{2} \frac{\partial}{\partial q_{1}}\right\} \exp \left\{i \eta q_{1} \frac{\partial}{\partial q_{2}}\right\} .
\end{aligned}
$$

Следует отметить, что выбор оператора $\widehat{S}$ в виде (8) производится в результате анализа возможности диагонализации стационарного уравнения Шредингера с гамильтонианом (5). В выражении (8) постоянные величины $\xi_{1}, \xi_{2}, \xi, \eta, \lambda_{1}, \lambda_{2}$ являются неизвестными, мы найдем их ниже.

Теперь наша задача заключается в нахождении таких $\xi_{1}, \xi_{2}, \xi, \eta, \lambda_{1}, \lambda_{2}$, чтобы гамильтониан $\widehat{H}^{\prime}$ в уравнении (7) стал диагональным. Для этого введем вспомогательный гамильтониан

$$
\widehat{\widetilde{H}}=\exp \left\{-i \xi q_{2} \frac{\partial}{\partial q_{1}}\right\} \exp \left\{i \eta q_{1} \frac{\partial}{\partial q_{2}}\right\} \widehat{H} \exp \left\{-i \eta q_{1} \frac{\partial}{\partial q_{2}}\right\} \exp \left\{i \xi q_{2} \frac{\partial}{\partial q_{1}}\right\} .
$$

Подберем такие значения $\xi, \eta$, чтобы "перекрестных" значений по $q_{1}$ и $q_{2}$ не было (т. е. чтобы пропало произведение $q_{1} q_{2}$ ):

$$
\xi=\frac{i}{2} \sqrt{\frac{\omega_{1}}{\omega_{2}}} \frac{\gamma}{\sqrt{\alpha^{2}+\gamma^{2}}}, \quad \eta=i \sqrt{\frac{\omega_{2}}{\omega_{1}}} \frac{\sqrt{\alpha^{2}+\gamma^{2}}-\alpha}{\gamma} .
$$

Проведя необходимые выкладки, получим

$$
\widehat{\widetilde{H}}=-\frac{1}{2} \Delta-i q_{1} \boldsymbol{\nabla} \mathbf{S}-i q_{2} \boldsymbol{\nabla} \boldsymbol{\Sigma}+q_{1}^{2} W^{2}+q_{2}^{2} \Lambda^{2}-V^{2} \frac{\partial^{2}}{\partial q_{1}^{2}}-L^{2} \frac{\partial^{2}}{\partial q_{2}^{2}},
$$


где

$$
\begin{aligned}
& \boldsymbol{\Sigma}=\frac{1}{2} \sqrt{\frac{\omega_{1}}{\omega_{2}}} \frac{\gamma \beta_{1}}{\sqrt{\alpha^{2}+\gamma^{2}}} \mathbf{u}_{1}+\frac{\beta_{2}}{2}\left(1+\frac{\alpha}{\sqrt{\alpha^{2}+\gamma^{2}}}\right) \mathbf{u}_{2}, \\
& \mathbf{S}=\beta_{1} \mathbf{u}_{1}-\sqrt{\frac{\omega_{2}}{\omega_{1}}} \beta_{2} \frac{\sqrt{\alpha^{2}+\gamma^{2}}-\alpha}{\gamma} \mathbf{u}_{2}, \\
& W^{2}=\frac{\omega_{2}}{\omega_{1}}\left(\omega_{2}+\beta_{2}^{2}\right)\left(1+\left(\frac{\alpha}{\gamma}\right)^{2}-\frac{\alpha \sqrt{\alpha^{2}+\gamma^{2}}}{\gamma^{2}}\right)-\frac{\sqrt{\alpha^{2}+\gamma^{2}}}{2}, \\
& \Lambda^{2}=\frac{1}{4}\left(\omega_{2}+\beta_{2}^{2}\right)\left(1+\frac{\alpha}{\sqrt{\alpha^{2}+\gamma^{2}}}\right)+\frac{1}{8} \frac{\omega_{1}}{\omega_{2}} \frac{\gamma^{2}}{\sqrt{\alpha^{2}+\gamma^{2}}}, \\
& V^{2}=\frac{\omega_{1}}{4}\left(1+\frac{\alpha}{\sqrt{\alpha^{2}+\gamma^{2}}}\right), \quad L^{2}=\omega_{2}\left(1+\left(\frac{\alpha}{\gamma}\right)^{2}\left(1-\frac{\sqrt{\alpha^{2}+\gamma^{2}}}{\alpha}\right)\right) \text {, } \\
& \alpha=\frac{\omega_{2}}{\omega_{1}}\left(\omega_{2}+\beta_{2}^{2}\right)-\left(\omega_{1}+\beta_{1}^{2}\right), \quad \gamma=2 \sqrt{\frac{\omega_{2}}{\omega_{1}}} \beta_{1} \beta_{2} \mathbf{u}_{1} \mathbf{u}_{2} .
\end{aligned}
$$

Далее найдем гамильтониан

$$
\begin{aligned}
\widehat{H}^{\prime}= & \exp \left\{i \xi_{2} \frac{\partial}{\partial x} \frac{\partial}{\partial q_{2}}+i \lambda_{1} \frac{\partial}{\partial z} \frac{\partial}{\partial q_{2}}+i \xi_{1} \frac{\partial}{\partial z} \frac{\partial}{\partial q_{1}}\right\} \exp \left\{-i \lambda_{2} x \frac{\partial}{\partial z}\right\} \widehat{\widetilde{H}} \times \\
& \times \exp \left\{i \lambda_{2} x \frac{\partial}{\partial z}\right\} \exp \left\{-i \xi_{2} \frac{\partial}{\partial x} \frac{\partial}{\partial q_{2}}-i \lambda_{1} \frac{\partial}{\partial z} \frac{\partial}{\partial q_{2}}-i \xi_{1} \frac{\partial}{\partial z} \frac{\partial}{\partial q_{1}}\right\} .
\end{aligned}
$$

Выбрав неизвестные параметры $\xi_{1}, \xi_{2}, \lambda_{1}, \lambda_{2}$ таким образом, чтобы гамильтониан $\widehat{H}^{\prime}$ стал диагональным, получим

$$
\begin{aligned}
\widehat{H}^{\prime}= & -\frac{1}{2} \Delta+q_{1}^{2} W^{2}+q_{2}^{2} \Lambda^{2}-V^{2} \frac{\partial^{2}}{\partial q_{1}^{2}}-L^{2} \frac{\partial^{2}}{\partial q_{2}^{2}}+ \\
& +\left(\frac{S^{2}}{4 W^{2}}+\frac{1}{2} \frac{(\boldsymbol{\Sigma} \mathbf{S} / S)^{2}}{2 \Lambda^{2}-((\boldsymbol{\Sigma} \times \mathbf{S}) / S)^{2}}\right) \frac{\partial^{2}}{\partial z^{2}}+\left(\frac{\boldsymbol{\Sigma} \times \mathbf{S}}{S}\right)^{2} \frac{1}{4 \Lambda^{2}} \frac{\partial^{2}}{\partial x^{2}}
\end{aligned}
$$

при этом $\xi_{1}, \xi_{2}, \lambda_{1}, \lambda_{2}$ выбирались в виде

$$
\begin{aligned}
\xi_{1} & =\frac{S}{2 W^{2}}, & \xi_{2} & =\frac{|\boldsymbol{\Sigma} \times \mathbf{S}|}{2 S \Lambda^{2}}, \\
\lambda_{1} & =\frac{\boldsymbol{\Sigma} \mathbf{S}}{S} \frac{1}{2 \Lambda^{2}-(\boldsymbol{\Sigma} \times \mathbf{S} / S)^{2}}, & \lambda_{2} & =-i \frac{1}{S^{2}} \frac{|\boldsymbol{\Sigma} \times \mathbf{S}| \boldsymbol{\Sigma} \mathbf{S}}{2 \Lambda^{2}-(\boldsymbol{\Sigma} \times \mathbf{S} / S)^{2}} .
\end{aligned}
$$

Решить уравнение Шредингера с гамильтонианом (13) не представляет труда, поскольку все переменные разделены, и эти решения имеют вид плоской волны и волновых функций гармонического осциллятора. Сначала запишем собственное значение энергии гамильтониана (13):

$$
\begin{aligned}
E_{\mathbf{k}, m, n}= & \frac{k_{x}^{2}}{2}\left(1-\frac{(\boldsymbol{\Sigma} \times \mathbf{S})^{2}}{2 S^{2} \Lambda^{2}}\right)+\frac{k_{z}^{2}}{2}\left(1-\left(\frac{S^{2}}{2 W^{2}}+\frac{1}{S^{2}} \frac{(\boldsymbol{\Sigma} \mathbf{S})^{2}}{2 \Lambda^{2}-((\boldsymbol{\Sigma} \times \mathbf{S}) / S)^{2}}\right)\right)+ \\
& +\frac{k_{y}^{2}}{2}+2 V W\left(m+\frac{1}{2}\right)+2 L \Lambda\left(n+\frac{1}{2}\right),
\end{aligned}
$$


где $k_{x}, k_{y}, k_{z}$ - проекции волнового вектора свободной частицы $\mathbf{k}$ на соответствующие оси координат, а $n, m=0,1,2, \ldots$ - квантовые числа. Далее выпишем собственную волновую функцию гамильтониана (13):

$$
\Psi_{\mathbf{k}, m, n}^{\prime}=C_{\mathbf{k}} e^{i \mathbf{k r}} C_{n} e^{-(\Lambda / L)\left(q_{2}^{2} / 2\right)} H_{n}\left(q_{2} \sqrt{\frac{\Lambda}{L}}\right) C_{m} e^{-(W / V)\left(q_{1}^{2} / 2\right)} H_{m}\left(q_{1} \sqrt{\frac{W}{V}}\right)
$$

где $C_{\mathbf{k}}$ - нормировочный множитель для волновой функции электрона, $H_{n}-$ полиномы Эрмита, а нормировочные волновые функции для электромагнитных полей

$$
C_{n}=\frac{1}{\sqrt{2^{n} n ! \sqrt{\pi}}}\left(\frac{\Lambda}{L}\right)^{-1 / 4}, \quad C_{m}=\frac{1}{\sqrt{2^{m} m ! \sqrt{\pi}}}\left(\frac{W}{V}\right)^{-1 / 4} .
$$

Для нахождения волновой функции $\Psi$ нужен оператор $\widehat{S}^{-1}$, который известен, поскольку известны все параметры в операторе $\widehat{S}$. Подействовав оператором $\widehat{S}^{-1}$ на волновую функцию $\Psi^{\prime}$, достаточно просто получить искомую волновую функцию, поскольку после действия $\widehat{S}^{-1}$ на волновую функцию электрона получим операторы смещения. В итоге получим

$$
\begin{aligned}
\Psi_{\mathbf{k}, m, n}= & C_{\mathbf{k}} C_{n} C_{m} e^{-\lambda_{2} k_{z} x+i \mathbf{k r}} \exp \left(-\frac{\Lambda}{2 L}\left(q_{2}+\lambda_{1} k_{z}+\xi_{2} k_{x}-i \eta q_{1}\right)^{2}\right) \times \\
& \times H_{n}\left(\left(q_{2}+\lambda_{1} k_{z}+\xi_{2} k_{x}-i \eta q_{1}\right) \sqrt{\frac{\Lambda}{L}}\right) \times \\
& \times \exp \left(-\frac{W}{2 V}\left(q_{1}(1+\xi \eta)+\xi_{1} k_{z}+i \xi q_{2}\right)^{2}\right) \times \\
& \times H_{m}\left(\left(q_{1}(1+\xi \eta)+\xi_{1} k_{z}+i \xi q_{2}\right) \sqrt{\frac{W}{V}}\right) .
\end{aligned}
$$

Из выражения (18) видно, что состояние системы, описываемой гамильтонианом (5), нельзя выразить через состояния с заданным числом “чистых" фотонов. Кроме того, полученный результат не может быть выражен через состояние "одетых" фотонов одномодового электромагнитного поля [9]. Также из общего вида выражения (18) следует, что нельзя выделить какую-либо частицу отдельно, и поэтому все частицы этой системы формально взаимодействуют друг с другом.

\section{4. ИОНИЗАЦИЯ АТОМА}

При расчете ионизации атома скрещенным лазерным полем необходимо знание следующих параметров системы: средние значения квадратов модулей амплитуд напряженности первого и второго лазерных полей $-\epsilon_{1}^{2}$ и $\epsilon_{2}^{2}$. Также нужно выписать закон сохранения энергии, который необходим при расчете скорости ионизации (6).

Определим напряженности поля (см., например, [9]):

$$
\omega_{1} m_{0}=\frac{\epsilon_{1}^{2}}{8 \pi} V_{f}, \quad \omega_{2} n_{0}=\frac{\epsilon_{2}^{2}}{8 \pi} V_{f}
$$

где $m_{0}, n_{0}$ - число фотонов в первом и втором поле соответственно. Тогда получим, что постоянные $\beta_{1}$ и $\beta_{2}$, которые присутствуют в выражении (18), можно выразить 
через число фотонов и напряженность поля в виде

$$
\beta_{1}=\sqrt{\frac{\epsilon_{1}^{2}}{2 \omega_{1}^{2} m_{0}}}, \quad \beta_{2}=\sqrt{\frac{\epsilon_{2}^{2}}{2 \omega_{2}^{2} n_{0}}} .
$$

Выражения (20) необходимы в таком виде, поскольку в (18) пропадает зависимость от $V_{f}$.

Далее рассмотрим разность $E_{\mathrm{f}}-E_{\mathrm{i}}$ в $(6)$, где $E_{\mathrm{i}}=-E_{\delta}+\omega_{1}\left(m_{0}+1 / 2\right)+\omega_{1}\left(n_{0}+1 / 2\right)$, a $E_{\mathrm{f}}$ определяется выражением (15), где $E_{\delta}=\left|E_{\delta}\right|$ - энергия электрона в атоме. В большинстве задач при взаимодействии сильного электромагнитного поля с веществом величины $n_{0}, m_{0}$ следует считать большими, $n_{0} \gg 1$ и $m_{0} \gg 1$ [9], поэтому все расчеты далее будут выполняться при этих условиях. Рассмотрим $E_{\mathrm{f}}-E_{\mathrm{i}}$, считая, что $n=n_{0}-l$, а $m=m_{0}-L$, где $l, L-$ количество поглощенных фотонов. Отметим, что о поглощенных фотонах в этой задаче нужно говорить условно, так как в начальном состоянии $n_{0}, m_{0}$ речь идет о "чистых" фотонах, а в конечном состоянии $n, m$ - об "одетых". Значения $L$ и $l$ задают все возможные процессы, разрешенные законом сохранения энергии, и, суммируя по $L$ и $l$, можно получить полную скорость ионизации. Покажем, что даже при $n_{0} \rightarrow \infty, m_{0} \rightarrow \infty$ разность энергий $E_{\mathrm{f}}-E_{\mathrm{i}}$ будет конечной величиной (хотя по отдельности величины $E_{\mathrm{f}}$ и $E_{\mathrm{i}}$ стремятся к бесконечности). Например, для одномодового поля в работе [9] показано, что итоговый результат для скорости ионизации при $n_{0} \gg 1$ не зависит от $n_{0}$. Рассмотрим два случая, которые интересны при исследовании ионизации атома: 1) два одинаковых скрещенных лазерных поля при $\alpha \ll \gamma ; 2)$ скрещенные лазерные поля при $\alpha \gg \gamma$. Остальные случаи рассматривать не будем, поскольку скорость ионизации зависит от числа фотонов в поле, а нас интересует случай, когда мы знаем только частоту лазерного поля и его напряженность. При $\alpha \gg \gamma$ также можно рассмотреть случай двух одинаковых скрещенных полей, но при этом считать, что $\omega_{1} \approx \omega_{2} \approx \omega$.

В первом случае одинаковых скрещенных полей имеем $\omega_{1}=\omega_{2}=\omega, \epsilon_{1}^{2}=\epsilon_{2}^{2}=\epsilon^{2}$, $n_{0}=m_{0}=s$. В итоге закон сохранения энергии имеет вид

$$
E_{\mathrm{f}}-E_{\mathrm{i}}=\frac{k^{2}}{2}+E_{\delta}+\frac{\epsilon^{2}}{2 \omega^{2}}-\omega(L+l),
$$

а основные параметры для этого случая

$$
\begin{gathered}
\alpha=0, \quad \beta_{1}=\beta_{2}=\beta, \quad \gamma=2 \beta^{2} \mathbf{u}_{1} \mathbf{u}_{2}, \quad \Lambda=\frac{\sqrt{\omega}}{2}, \quad L=\sqrt{\omega}, \\
W=\sqrt{\omega}, \quad V=\frac{\sqrt{\omega}}{2}, \quad \xi_{1}=\frac{\beta}{\sqrt{2} \omega} \sqrt{1-\left|\mathbf{u}_{1} \mathbf{u}_{2}\right|}, \quad \xi_{2}=\frac{\sqrt{2} \beta}{\omega} \sqrt{1+\left|\mathbf{u}_{1} \mathbf{u}_{2}\right|}, \\
\boldsymbol{\Sigma S}=0, \quad \xi=\frac{i}{2} \frac{\gamma}{|\gamma|}, \quad \eta=i \frac{\gamma}{|\gamma|} .
\end{gathered}
$$

Во втором случае, когда $\alpha \gg \gamma$, закон сохранения энергии имеет вид

$$
E_{\mathrm{f}}-E_{\mathrm{i}}=\frac{k^{2}}{2}+E_{\delta}+\frac{\epsilon_{1}^{2}}{4 \omega_{1}^{2}}+\frac{\epsilon_{2}^{2}}{4 \omega_{2}^{2}}-\omega_{1} L-\omega_{2} l,
$$


а основные параметры -

$$
\begin{aligned}
& \boldsymbol{\Sigma}=\beta_{2} \mathbf{u}_{2}, \quad \mathbf{S}=\beta_{1} \mathbf{u}_{1}, \quad W^{2}=\frac{\omega_{1}}{2}, \quad \Lambda^{2}=\frac{\omega_{2}}{2}, \quad V^{2}=\frac{\omega_{1}}{2}, \quad L^{2}=\frac{\omega_{2}}{2}, \\
& \xi_{1}=\frac{\beta_{1}}{\omega_{1}}, \quad \xi_{2}=\beta_{2} \frac{\left|\mathbf{u}_{1} \times \mathbf{u}_{2}\right|}{\omega_{2}}, \quad \lambda_{1}=\frac{\beta_{2} \mathbf{u}_{1} \mathbf{u}_{2}}{\omega_{2}}, \quad \lambda_{2}=\frac{\beta_{2}^{2} \mathbf{u}_{1} \mathbf{u}_{2}\left|\mathbf{u}_{1} \times \mathbf{u}_{2}\right|}{\omega_{2}} .
\end{aligned}
$$

Далее выпишем матричный элемент в общем виде для рассмотренных случаев:

$$
T_{\mathrm{f}, \mathrm{i}}=F(\mathbf{k})\left\langle\Phi_{\mathbf{k}, m, n}|\hat{I}| \Phi_{0}\right\rangle
$$

где оператор взаимодействия

$$
\begin{aligned}
\hat{I} & =\left(\beta_{1} q_{1} \mathbf{u}_{1}+\beta_{2} q_{2} \mathbf{u}_{2}\right) \mathbf{k}+\frac{1}{2}\left(\beta_{1} q_{1} \mathbf{u}_{1}+\beta_{2} q_{2} \mathbf{u}_{2}\right)^{2}, \\
F(\mathbf{k}) & =C_{\mathbf{k}} \int e^{-i \mathbf{k r}} \phi_{0}(\mathbf{r}) d^{3} \mathbf{r} .
\end{aligned}
$$

В случае скрещенных двух одинаковых лазерных полей

$$
\begin{aligned}
\Phi_{\mathbf{k}, m, n}= & C_{n} C_{m} \exp \left[-\left(\frac{q_{1}}{2}-\frac{1}{2} \frac{\gamma}{|\gamma|} q_{2}+\xi_{1} k_{z}\right)^{2}\right] \times \\
& \times \exp \left[-\frac{1}{4}\left(q_{2}+\frac{\gamma}{|\gamma|} q_{1}+\xi_{2} k_{x}\right)^{2}\right] \times \\
& \times H_{m}\left(\sqrt{2}\left(\frac{q_{1}}{2}-\frac{1}{2} \frac{\gamma}{|\gamma|} q_{2}+\xi_{1} k_{z}\right)\right) H_{n}\left(\frac{1}{\sqrt{2}}\left(q_{2}+\frac{\gamma}{|\gamma|} q_{1}+\xi_{2} k_{x}\right)\right), \\
\Phi_{0}= & C_{s}^{2} e^{-q_{1}^{2} / 2} e^{-q_{2}^{2} / 2} H_{s}\left(q_{1}\right) H_{s}\left(q_{2}\right) .
\end{aligned}
$$

В случае $\alpha \gg \gamma$ имеем

$$
\begin{aligned}
\Phi_{\mathbf{k}, m, n}= & \exp \left[-\frac{1}{2}\left(q_{1}+\frac{\beta_{1}}{\omega_{1}} k_{z}-\frac{\gamma}{2 \alpha} \sqrt{\frac{\omega_{1}}{\omega_{2}}} q_{2}\right)^{2}\right] \times \\
& \times \exp \left[-\frac{1}{2}\left(q_{2}+k_{z} \lambda_{1}+k_{x} \xi_{2}+\frac{\gamma}{2 \alpha} \sqrt{\frac{\omega_{2}}{\omega_{1}}} q_{1}\right)^{2}\right] \times \\
& \times H_{m}\left(q_{1}+\frac{\beta_{1}}{\omega_{1}} k_{z}-\frac{\gamma}{2 \alpha} \sqrt{\frac{\omega_{1}}{\omega_{2}}} q_{2}\right) \times \\
& \times H_{n}\left(q_{2}+k_{z} \lambda_{1}+k_{x} \xi_{2}+\frac{\gamma}{2 \alpha} \sqrt{\frac{\omega_{2}}{\omega_{1}}} q_{1}\right) \\
\Phi_{0}= & C_{n_{0}} C_{m_{0}} e^{-q_{1}^{2} / 2} e^{-q_{2}^{2} / 2} H_{m_{0}}\left(q_{1}\right) H_{n_{0}}\left(q_{2}\right) .
\end{aligned}
$$

Матричные элементы (25) с волновыми функциями (27) и (28) не вычисляются в аналитическом виде. Численный расчет этих выражений и нахождение приближенных аналитических формул для (25) в настоящей работе не проводились. Заметим, что в выражении (28) нельзя отбросить какой-либо из членов, например $(\gamma / 2 \alpha) q_{1}$, несмотря на то что $\gamma \sim 1 / \sqrt{n m} \ll 1$ при $n \gg 1$ и $m \gg 1$. Это связано с тем, что основной вклад в интеграл $(25)$ вносят $q_{1} \sim \sqrt{m}$ и $q_{2} \sim \sqrt{n}$, что в итоге ведет к равнозначности всех членов. 


\section{5. ЗАКЛЮЧЕНИЕ}

В работе решено уравнение Шредингера для электрона в двухмодовом квантованном электромагнитном поле. Полученное решение позволяет проводить расчеты ионизации атома в двух скрещенных лазерных полях большой интенсивности. Точное решение (18) уравнения Шредингера с гамильтонианом (5) важно не только для теории ионизации, но и с фундаментальной точки зрения, поскольку поведение сложной квантовой системы представлено в аналитическом виде. Кроме того, полученный результат (18) указывает на характер связи рассмотренной системы, в которой все частицы формально взаимодействуют друг с другом. Отметим, что спин электрона не вносит значимой поправки в нерелятивистском случае и его не учитывают [1]-[3]. Действительно, энергия электрона в электромагнитном поле пропорциональна напряженности электромагнитного поля $E$ (в дипольном случае в калибровке “длины” [4], [8]), и энергия, связанная с действием электромагнитного поля на спин частицы, пропорциональна $E / c$, что пренебрежимо мало.

\section{Список литературы}

[1] Л.В. Келдыш, "Ионизация в поле сильной электромагнитной волны”, ЖЖТФ, 47:5 (1965), 1945-1957.

[2] В.С. Попов, "Туннельная и многофотонная ионизация атомов и ионов в сильном лазерном поле (теория Келдыша)", УФН, 174:9 (2004), 921-951.

[3] Н. Б. Делоне, В. П. Крайнов, "Туннельная и надбарьерная ионизация атомов и ионов в поле лазерного излучения", УФН, 168:5 (1998), 531-549.

[4] Б. М. Карнаков, В. Д. Мур, С.В.Попруженко, В. С. Попов, "Современное развитие теории нелинейной ионизации атомов и ионов", УФН, 185:1 (2015), 3-34.

[5] V. Yanovsky, V. Chvykov, G. Kalinchenko, P. Rousseau, T. Planchon, T. Matsuoka, A. Maksimchuk, J. Nees, G. Cheriaux, G. Mourou, K. Krushelnick, "Ultra-high intensity300-TW laser at $0.1 \mathrm{~Hz}$ repetition rate", Opt. Express, 16:3 (2008), 2109-2114.

[6] F. Krausz, M. Ivanov, "Attosecond physics", Rev. Modern Phys., 81:1 (2009), 163-234.

[7] Л. Мандель, Э. Вольф, Оптическая когерентность и квантовая оптика, Наука, М., 2000.

[8] М. О. Скалли, М. С. Зубайри, Квантовая оптика, Физматлит, М., 2003.

[9] И. А. Гоносков, Г. А. Вугальтер, В. А. Миронов, "Ионизация в квантованном электромагнитном поле", ЖЖТФ, 132:6 (2007), 1278-1290.

[10] И. А. Буренков, О.В. Тихонова, "Ионизация и стабилизация атома в квантовом электромагнитном поле", Писъма в ЖКЭТФ, 97:6 (2013), 409-414.

[11] И. Берсон, “Электрон в квантованном поле монохроматической электромагнитной волны”, ЖЭТФ, 56:5 (1969), 1627-1633.

[12] D. S. Guo, T. Aberg, "Quantum electrodynamical approach to multiphoton ionisation in the high-intensity $H$ field", J. Phys. A: Math. Cen., 21:24 (1988), 4577-4585.

[13] D. S. Guo, G. W. F. Drake, "Stationary solutions for an electron in an intense laser field. I. Single-mode case", J. Phys. A: Math. Cen., 25:11 (1992), 3383-3397.

Поступила в редакцию 24.01.2016, после доработки 23.03.2016 